\title{
Metaanálisis comparativo de hernioplastia inguinal TAPP vs Lichtenstein
}

\section{A comparative meta-analysis of inguinal hernioplasty TAPP vs Lichtenstein}

\author{
Federico Chávez Peón Pérez, ${ }^{*}$ José Ignacio Rodríguez Arias, ${ }^{\ddagger}$ Tomás Barrientos Fortes ${ }^{\S}$
}

Citar como: Chávez PPF, Rodríguez AJI, Barrientos FT. Metaanálisis comparativo de hernioplastia inguinal TAPP vs Lichtenstein. Acta Med. 2020; 18 (4): 373-381. https://dx.doi.org/10.35366/97263

\section{Resumen}

El metaanálisis compara el abordaje laparoscópico transabdominal preperitoneal contra la reparación tipo Lichtenstein. Se tomaron en cuenta ensayos clínicos aleatorizados, utilizando la metodología PRISMA para la inclusión y exclusión de los estudios filtrados, los datos de los estudios seleccionados se agruparon en diagramas de Forest Plot. Se utilizaron 13 ensayos clínicos controlados aleatorizados (11 grupos de pacientes) con un total de 1,172 pacientes. El tiempo quirúrgico favorece al grupo $\mathrm{TL},\left(\mathrm{IC}_{95 \%}, 9.67-34.03, \mathrm{p}<0.00001\right)$. No existe diferencia significativa en cuanto al tipo de estancia hospitalaria. Hubo nueve casos más de infección en el grupo LT, (IC ${ }_{95 \%}$, OR 0.26, [0.07, 0.99], p = 0.05). No existió diferencia significativa en cuanto a la presentación de hematoma en ambos grupos. El dolor crónico favoreció significativamente al grupo TAPP, (IC ${ }_{95 \%}$, OR $\left.0.32[0.13,0.82], p=0.02\right)$. La recurrencia de hernia inguinal no tuvo diferencia significativa entre los grupos. El dolor agudo postoperatorio favoreció al grupo TAPP en las primeras 12 y 24 horas, posteriormente no hubo diferencia estadísticamente significativa. El tiempo quirúrgico fue significativamente más corto en el caso del abordaje abierto. El abordaje laparoscópico presentó muchas menos complicaciones respecto a infección y dolor crónico.

Palabras clave: Hernia inguinal, Lichtenstein, transabdominal preperitoneal.

\section{Abstract}

A meta-analysis comparing the laparoscopic transabdominal preperitoneal approach versus the Lichtenstein-type repair was performed. Randomized clinical trials were taken into account, using the PRISMA methodology for the inclusion and exclusion of the filtered studies, the data of the selected studies were grouped in Forest Plot diagrams. Thirteen randomized controlled clinical trials (11 groups of patients) were found which compared LT against TAPP in inguinal hernioplasty, a total of 1,172 patients. Regarding the surgical time, the TL group is favored $(95 \% \mathrm{Cl}, 9.67-34.03, \mathrm{p}<0.00001)$. There is no significant difference in the type of hospital stay. In the case of infection, there were nine more cases in the LT group $(95 \% \mathrm{Cl}$, OR 0.26 , $[0.07,0.99], p=0.05)$. There was no significant difference in the presentation of hematoma in both groups. Chronic pain significantly favored the TAPP group $(95 \% \mathrm{Cl}$, OR 0.32 [0.13, 0.82 ], $p=0.02$ ). Regarding the recurrence of inguinal hernia there was no significant difference between the groups. Acute postoperative pain favored the TAPP group in the first 12 and 24 hours, on the later hours there was no statistically significant difference. The surgery duration time was significantly shorter in the open group. Laparoscopic approach resulted with lesser complications in regards to infection and chronic pain.

Keywords: Inguinal hernia, Lichtenstein, pre-peritoneal transabdominal.

\section{INTRODUCCIÓN}

* Instituto Mexicano de Neurociencias. Facultad de Ciencias de la Salud, Universidad Anáhuac México.

* Facultad de Ciencias de la Salud, Universidad Anáhuac México.

$\S$ Fellow of the American College of Surgeons. Cirujano, Hospital Ángeles Lomas.

Correspondencia:

Dr. Tomás Barrientos Fortes

Correo electrónico: tbarrien@anahuac.mx

Aceptado: 25-03-2020.

www.medigraphic.com/actamedica

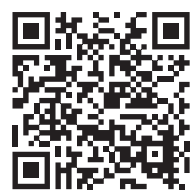

En México y en el mundo la incidencia de hernia inguinal está calculada entre 3 y $5 \%{ }^{1}$ Su historia se remonta a registros de los médicos sumerios en Mesopotamia 4,000 años a.C. donde aparece el concepto de "herniotomía". ${ }^{2}$ A lo largo del tiempo se han desarrollado técnicas que refuerzan las paredes del conducto inguinal, con resultados muy variables entre las técnicas. Las técnicas que se desarrollan actualmente bajo el concepto "libre de tensión" utilizan malla sintética y han dado los mejores resultados hasta el momento. ${ }^{3}$ Ger describe en 1982 la primera reparación 
herniaria laparoscópica, y en 1992 Arregui y colaboradores describen el procedimiento de reparación transabdominal preperitoneal. ${ }^{4}$

Aparte de las técnicas de reparación abierta convencionales, la cirugía laparoscópica de mínimo acceso es la técnica preferida por algunos autores. Sin embargo, el debate sobre la vía de abordaje que se debe realizar persiste. En Latinoamérica se calcula que solamente $8 \%$ de las hernioplastias se realizan con abordaje laparoscópico. ${ }^{5}$ Muchos de los estudios publicados que se orientan a la comparación de técnicas de reparación abierta contra el abordaje laparoscópico de mínimo acceso son estudios no aleatorizados. ${ }^{6}$ Otros estudios, incluyendo metaanálisis, demuestran poca o nula diferencia entre los beneficios de ambos abordajes. ${ }^{7-11}$

\section{MATERIAL Y MÉTODOS}

Se realizó una revisión sistemática de la literatura y un metaanálisis según los parámetros de Cochrane $^{12}$ para comparar los resultados de ambos abordajes quirúrgicos. Es un tipo de estudio documental, retrospectivo con enfoque cuantitativo. El propósito fue encontrar la información relevante sobre la TL, y TAPP para la reparación de hernias inguinales directas e indirectas. Se realizó la búsqueda de información en bancos digitales reconocidos (ClinicalKey, PubMed y Cochrane). Para el estudio se incluyeron los ensayos clínicos aleatorizados escritos en inglés (excepto Köeninger y colaboradores en 1998 en alemán, que pertenece a la misma serie de pacientes que los estudios de Köeninger y colaboradores y Butters y su equipo) $)^{13-15}$ a partir de 1992, año en el que se introdujo TAPP a la práctica quirúrgica. No se consideró el país de origen o el hospital de procedencia para la inclusión de la información. Para la búsqueda se utilizaron las palabras: hernia inguinal, Lichtenstein, TAPP y los operadores boléanos AND, OR y NOT.

Se incluyeron 13 ensayos clínicos controlados aleatorizados (11 grupos de pacientes), donde se comparaba directamente el abordaje TL contra la TAPP en pacientes con hernioplastia inguinal para la reparación de hernia inguinal directa o indirecta, unilateral o bilateral. Los artículos seleccionados tomaron en cuenta pacientes mayores de 18 años (ambos sexos), a quienes se les realizó hernioplastia con abordaje TL o con TAPP. Asimismo, los artículos excluían a todos aquellos pacientes mayores de 75 años, pacientes con hernias complicadas o recurrentes, pacientes que se tuvieron que intervenir de urgencia, y pacientes con hernias femorales.

Se utilizó la metodología PRISMA para la inclusión y exclusión de los estudios filtrados. Por medio de las palabras clave se buscaron e identificaron los artículos en las diferentes bases de datos. Se eliminaron los duplicados y los estudios que no cumplían con los criterios de inclusión o exclusión. Con los resultados alcanzados se analizó el texto completo para el análisis de la información y su inclusión en este metaanálisis. ${ }^{16}$

Se recuperó información sobre las características de cada estudio incluyendo año de publicación, país, número de pacientes que participaron en el estudio, abordajes quirúrgicos utilizados y seguimiento. Se investigó sobre las características de los pacientes incluidos en cada estudio en relación con su edad, sexo y tipo de hernia. Se analizaron los resultados de cada artículo en cuanto a número de cirujanos involucrados, su experiencia, técnica de aleatorización, dolor postoperatorio, dolor crónico, tiempo quirúrgico, tiempo de hospitalización, hematoma, recurrencia e infección. El dolor agudo y crónico fue extraído y reportado en la escala análoga visual del dolor (EVA), y se homologaron todos los resultados en una escala de 0-10. El dolor agudo postoperatorio se analizó en cuatro grupos por temporalidad. El grupo A reporta el dolor en las primeras 12 horas posoperatorias, el grupo B en las 12-24 horas, el grupo C de 24-48 horas y el grupo D de 48-72 horas postquirúrgicas.

Los datos de los estudios seleccionados se organizaron por variable analizada, agrupándolos en diagramas de forest plot. Estos diagramas muestran el peso de cada estudio representado por el tamaño del cuadro, la razón de momios o diferencia significativa estimada por la posición en el eje x y la línea horizontal representa el intervalo de confianza. Se excluyeron de cada grupo de análisis los artículos que no reportaban esa variable en particular.

Se analizaron variables dicotómicas y continuas, en el caso de las variables dicotómicas se calculó la razón de momios y en el caso de las variables continuas se calculó la diferencia significativa. En los estudios que reportan sus resultados con media y rango, se estimó la desviación estándar (DE) utilizando las fórmulas de Luo y colaboradores ${ }^{17}$ Además, se calculó la heterogeneidad del estudio utilizando la distribución de Pearson o $\chi^{2}$, estableciendo una significancia estadística de $p<0.05$. Se reportó el intervalo de confianza $\left(\mathrm{IC}_{95 \%}\right)$ para cada valor analizado. Todas las variables se analizaron bajo el modelo efectos aleatorios (random effects).

Para el análisis estadístico y la generación de forest plots se utilizó el programa Review Manager (RevMan 5), versión 5.3 Copenhagen: The Nordic Cochrane Centre, The Cochrane Collaboration, 2014.

\section{RESULTADOS}

En la búsqueda se identificaron 574 estudios. Mediante los criterios de inclusión y exclusión y las características de los artículos se seleccionaron 13 ensayos clínicos 
controlados aleatorizados (11 grupos de pacientes), los cuales comparaban directamente la TL contra la TAPP en la hernioplastia inguinal. ${ }^{13-15,18-27}$ Los dos artículos de Köeninger y colaboradores ${ }^{13,14}$ y el de Butters y colaboradores ${ }^{15}$ comparan al mismo grupo de pacientes en diferente año, por lo que se analizaron como un solo estudio, contando con un total de 11 grupos para el metaanálisis (Figura 1). Los datos generales de los ensayos seleccionados se resumen en la Tabla 1.
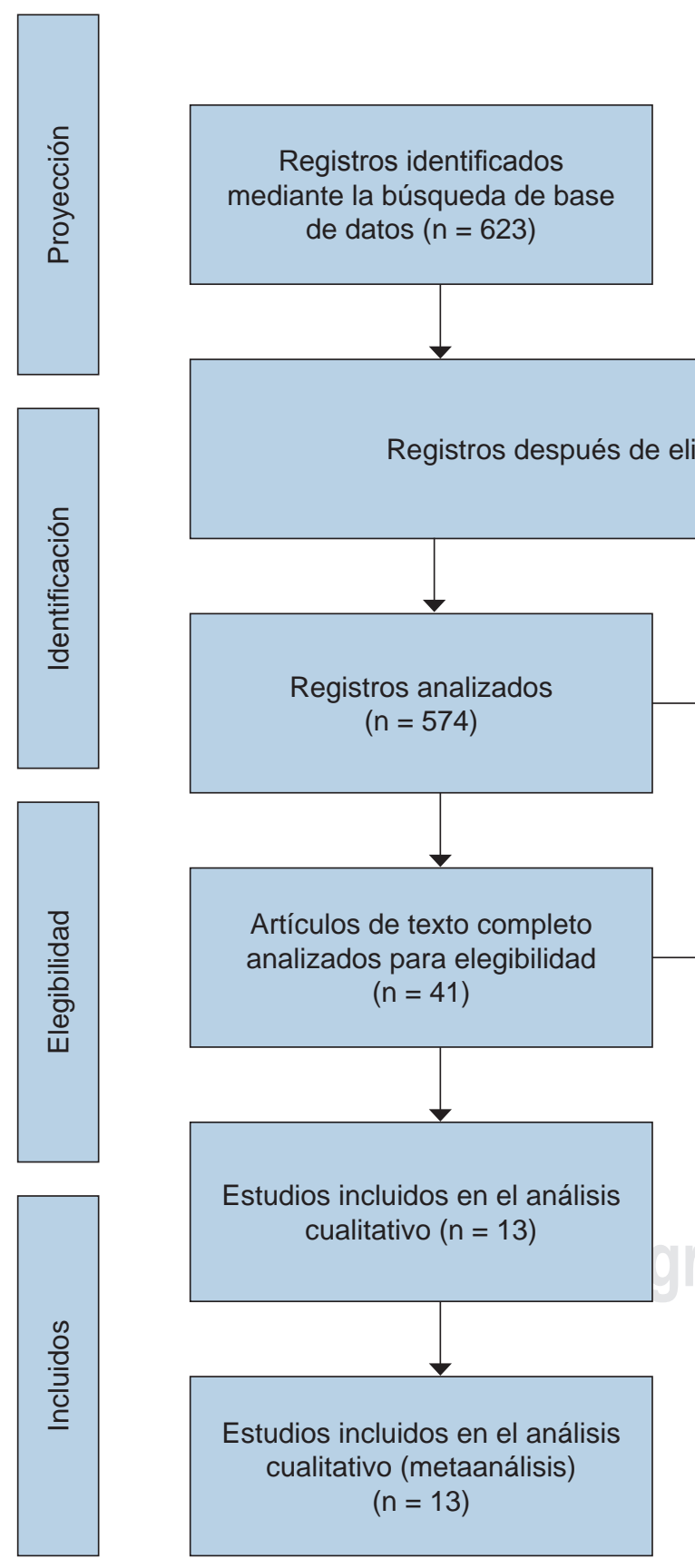

Figura 1:

Estrategia para la selección de publicaciones para el metaanálisis.
Este metaanálisis incluye un total de 1,172 pacientes, 585 en el grupo a los que se les realizó la TL y 587 en el grupo de la técnica TAPP. Respecto a los cirujanos que intervinieron a los pacientes, en siete artículos fueron realizadas por un grupo de cirujanos, ${ }^{14,18,20,23,25-27}$ en tres artículos por un solo cirujano, ${ }^{21,22,24}$ y en un artículo no refieren este dato. ${ }^{19}$ En cuanto a la experiencia de los cirujanos, siete artículos los reportan como experimentados, ${ }^{14,18,19,21,24-26}$ un artículo con moderada experiencia ${ }^{22}$
Registros adicionales encontrados en otras fuentes $(n=37)$

Registros excluidos por título y resumen $(n=534)$

Artículos de texto completo excluidos por diferente técnica quirúrgica $(n=14)$, hernia recurrente $(n=7)$, hernia complicada $(n=4)$, población estudiada $(n=3)$ 
Tabla 1: Análisis comparativo entre series de pacientes tratados con técnicas laparoscópicas y abiertas.

\begin{tabular}{|c|c|c|c|c|c|c|c|c|c|c|}
\hline \multirow[b]{2}{*}{ Autor } & \multirow[b]{2}{*}{ Año } & \multirow[b]{2}{*}{ País } & \multirow[b]{2}{*}{ Periodo } & \multicolumn{2}{|c|}{ Núm. pacientes } & \multicolumn{2}{|c|}{ Edad } & \multicolumn{2}{|c|}{$\begin{array}{c}\text { Sexo } \\
\text { masculino/femenino }\end{array}$} & \multirow[b]{2}{*}{$\begin{array}{l}\text { Seguimiento } \\
\text { (meses) }\end{array}$} \\
\hline & & & & Lichtenstein & TAPP & Lichtenstein & TAPP & Lichtenstein & TAPP & \\
\hline Abbas et al. ${ }^{18}$ & 2012 & Egipto & $\begin{array}{c}\text { Mayo 2008- } \\
\text { septiembre } 2011\end{array}$ & 97 & 88 & $34.62 \pm 11.18$ & $35.89 \pm 12.104$ & $94 / 3$ & $86 / 2$ & $17.9(8-30)$ \\
\hline Anadol et al. ${ }^{19}$ & 2004 & Turquía & N/A & 25 & 25 & $41.2 \pm 10.9$ & $41.8 \pm 10.8$ & $25 / 0$ & $25 / 0$ & $13.5(8-28)$ \\
\hline Benedetto et al. ${ }^{20}$ & 2017 & España & $2013-2015$ & 73 & 61 & $54.7(27-70)$ & $52(31-70)$ & $68 / 5$ & $48 / 2$ & 12 \\
\hline Hamza et al. ${ }^{21}$ & 2010 & Egipto & N/A & 25 & 25 & $35.12 \pm 10.11$ & $36.73 \pm 12.06$ & $25 / 0$ & $25 / 0$ & 24 semanas \\
\hline Heikkinen et al. ${ }^{22}$ & 1998 & Finlandia & $\begin{array}{c}\text { Diciembre } \\
\text { 1994-junio } 1995\end{array}$ & 20 & 18 & $55.5(26-29)$ & $51.0(34-68)$ & $20 / 0$ & $17 / 1$ & 17 \\
\hline Koju et al. ${ }^{23}$ & 2017 & Nepal & $\begin{array}{l}\text { Octubre 2012- } \\
\text { marzo } 2014\end{array}$ & 51 & 51 & $49.20 \pm 18.18$ & $44.27 \pm 15.04$ & N/A & N/A & 12 \\
\hline $\begin{array}{l}\text { Köninger et al, } \\
\text { Butters et al. }{ }^{13-15}\end{array}$ & $\begin{array}{l}1998,2004 \\
2007\end{array}$ & Alemania & 12 meses & 76 & 81 & $53(26-74)$ & $53(30-74)$ & $25 / 0$ & $25 / 0$ & $52(46-60)$ \\
\hline Nadim et al. ${ }^{24}$ & 2013 & Pakistán & $\begin{array}{l}\text { Enero 2011- } \\
\text { abril } 2012\end{array}$ & 50 & 50 & $35.0 \pm 11.07$ & $39.50 \pm 9.43$ & $40 / 10$ & $43 / 7$ & Seis semanas \\
\hline Picchio et al. ${ }^{25}$ & 1999 & Letonia & $\begin{array}{l}\text { Noviembre 1996- } \\
\text { diciembre } 1997\end{array}$ & 52 & 52 & $55.2 \pm 12.4$ & $57.7 \pm 11.0$ & $40 / 12$ & $37 / 15$ & Cuatro semana \\
\hline Pokorny et al. ${ }^{26}$ & 2008 & Austria & 1998-2002 & 69 & 93 & $52(19-84)$ & $49(21-78)$ & $64 / 5$ & $86 / 7$ & Hasta 36 \\
\hline Salma et al. ${ }^{27}$ & 2015 & Pakistán & $\begin{array}{l}\text { Enero 2009- } \\
\text { diciembre } 2009\end{array}$ & 30 & 30 & 61.4 & $8 \pm 7$ & $30 / 0$ & $30 / 0$ & Hospitalización \\
\hline
\end{tabular}

y tres artículos no lo reportan. ${ }^{20,23,27}$ La técnica de aleatorización de cada estudio varía, con cuatro artículos con asignación de números aleatorios, ${ }^{21,23,24,26}$ tres con aleatorización por computadora, 20,25,27 dos que utilizan sobres sellados, ${ }^{14,22}$ uno con asignación por pelotas numeradas ${ }^{18}$ y un artículo no lo reporta. ${ }^{19}$

En la Tabla $2 \mathrm{~A}$ se puede observar cómo en todos los estudios analizados las medias de tiempo quirúrgico fueron mayores en el caso de la cirugía laparoscópica (TAPP). En el cálculo se favorece al grupo TL por una diferencia significativa, 20.80 minutos menor que el grupo TAPP $\left(\mathrm{IC}_{95 \%}\right.$, 11.87-29.73, $p<0.00001)$. La mayoría de los estudios no reportan el periodo quirúrgico que tomaron en cuenta, es decir, si el tiempo contó desde que ingresa el paciente a quirófano, es sedado o inicia el procedimiento, tampoco definen cómo establecen el final de la cirugía.

Cinco estudios reportan tiempo de estancia hospitalaria (Tabla 2B). Otros estudios registran el tiempo de regreso al trabajo o a las actividades diarias, pero con metodologías distintas, por lo que no fueron tomados en cuenta, la mayoría de estos estudios reportan que el grupo TL tardaba más tiempo en retornar a las actividades diarias. En el caso de tiempo de hospitalización el rango fue de uno a 10 días de hospitalización. La diferencia media entre grupos es de
0.14 días $\left(\mathrm{IC}_{95 \%},-0.45,0.17, \mathrm{p}=0.37\right)$. Se desconocen los sistemas y/o servicios de salud a los que pertenecían los pacientes, así como protocolos y políticas hospitalarias de cada estudio.

De los 13 estudios incluidos sólo cinco reportan infección de la herida quirúrgica en su muestra (Tabla 3A). En el forest plot se puede observar la tendencia que favorece significativamente a TAPP, el cálculo muestra una razón de $0.26\left(\mathrm{IC}_{95 \%}, 0.07-0.99, \mathrm{p}=0.05\right)$.

Cinco estudios reportan hematoma en sus resultados (Tabla 3B). El grupo TL presentó un caso más de hematoma postquirúrgico (20 vs. 21). La razón de momios es de 0.26 favoreciendo a TAPP $\left(\mathrm{IC}_{95 \%}, 0.50-1.84\right)$, pero el resultado no es estadísticamente significativo $(p=0.91)$.

Se incluyeron en total seis estudios para el análisis del dolor agudo postoperatorio, ya que lo reportaban utilizando la escala análoga visual del dolor (EVA) en escala 0-10 (Tabla 4). Otros estudios utilizaban dosis y frecuencia de medicación, pero por la heterogeneidad de los resultados se excluyeron.

El grupo A (primeras 12 horas postoperatorias) se conformó por tres estudios (Tabla 4A). Se observa una diferencia media que favorece a TAPP de -1.52 (IC $\left.\mathrm{C}_{95 \%},-1.99,-1.05\right)$, valor $\mathrm{p} \leq 0.00001$. 
El grupo B (12-24 horas postquirúrgicas) agrupa a cinco estudios (Tabla 4B). La diferencia media es de -1.23 en favor de TAPP ( $\left(\mathrm{I}_{95 \%},-2.49,-0.03\right)$, valor $\mathrm{p} \leq 0.06$.

En cuanto al grupo C (24-48 horas) el análisis de tres estudios arroja una diferencia media de -0.36 en favor del grupo TAPP, $\left(\mathrm{IC}_{95 \%},-1.22,-0.51\right)$, valor $\mathrm{p}=0.42$ (Tabla 4C).

El último grupo (48-72 horas postoperatorias) sólo es descrito por dos estudios (Tabla 4D). En éstos la diferencia media es de -0.89 en favor de TAPP $\left(\mathrm{IC}_{95 \%},-2.01,-0.23\right)$, valor $\mathrm{p}=0.12$.

Cuatro estudios reportan dolor crónico (Tabla 5A). En el grupo TL se presentaron 15 eventos más que en el grupo TAPP (21 vs. seis). En el cálculo acumulado la razón de momios de 0.32 en favor de TAPP ( $\left.\mathrm{IC}_{95 \%}, 0.13-0.82\right)$, presenta un valor $p=0.02$.

En el grupo TAPP se presentaron 14 casos de recurrencia de hernia inguinal, mientras que en el grupo TL 15, esto en los cinco estudios que lo reportan (Tabla 5B). El forest plot muestra una tendencia que favorece al grupo TAPP, con una razón de momios de 0.92 ( IC $\left._{95 \%}, 0.42-2.00\right)$, valor $p=0.83$.

\section{DISCUSIÓN}

Un procedimiento quirúrgico cuenta con muchas variables y factores que dificultan el estudio y comparación entre los resultados de uno u otro abordaje. Es difícil realizar un ensayo controlado aleatorizado que tome en cuenta y controle todas estas variables como el tiempo, dolor, complicaciones, etc. Por esta razón, no toda la bibliografía encontrada medía las mismas variables. Se realizó esta revisión sistemática con el fin de analizar los resultados de estos ensayos. Se discutirá cada variable analizada a continuación:

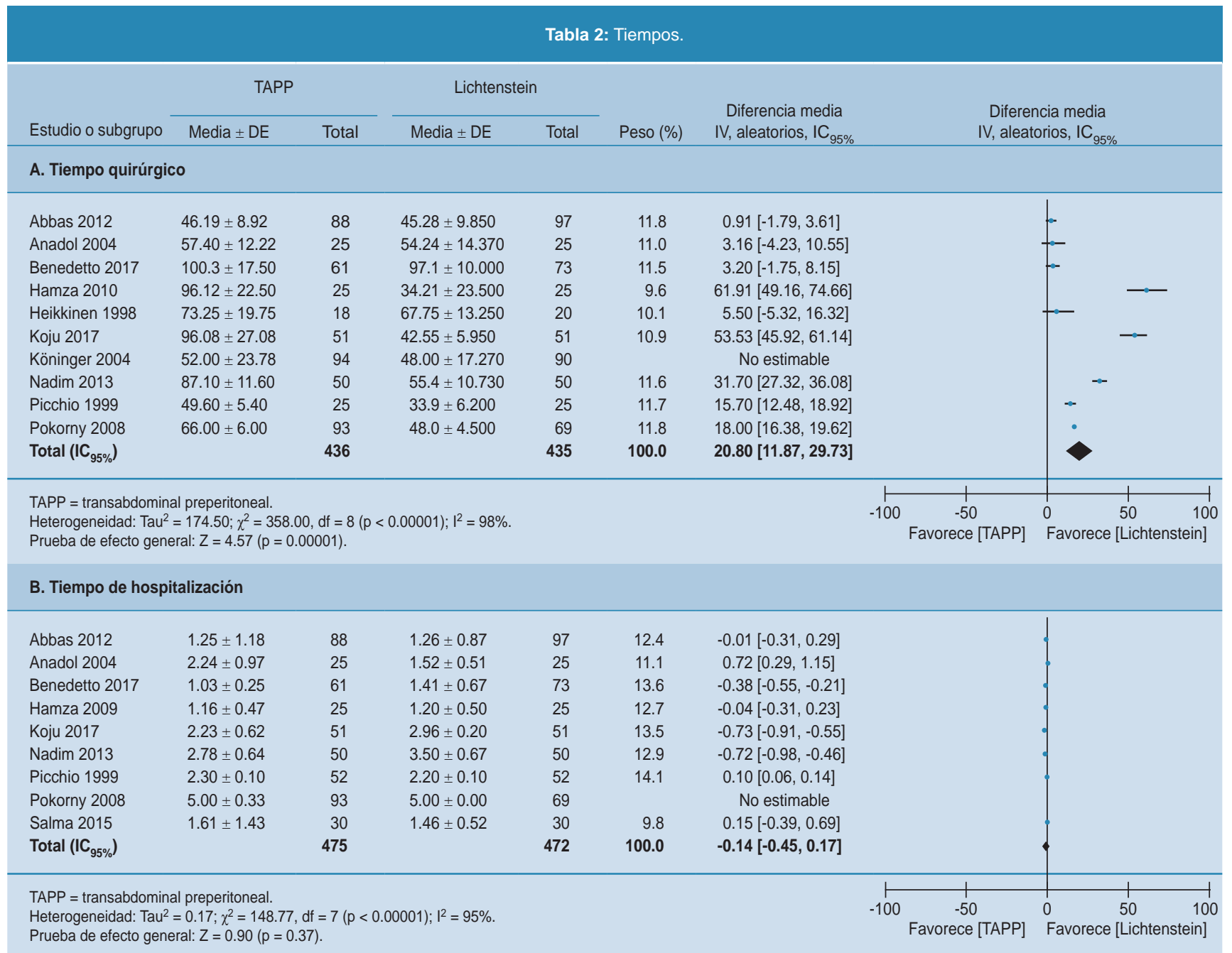


Tabla 3: Complicaciones agudas.

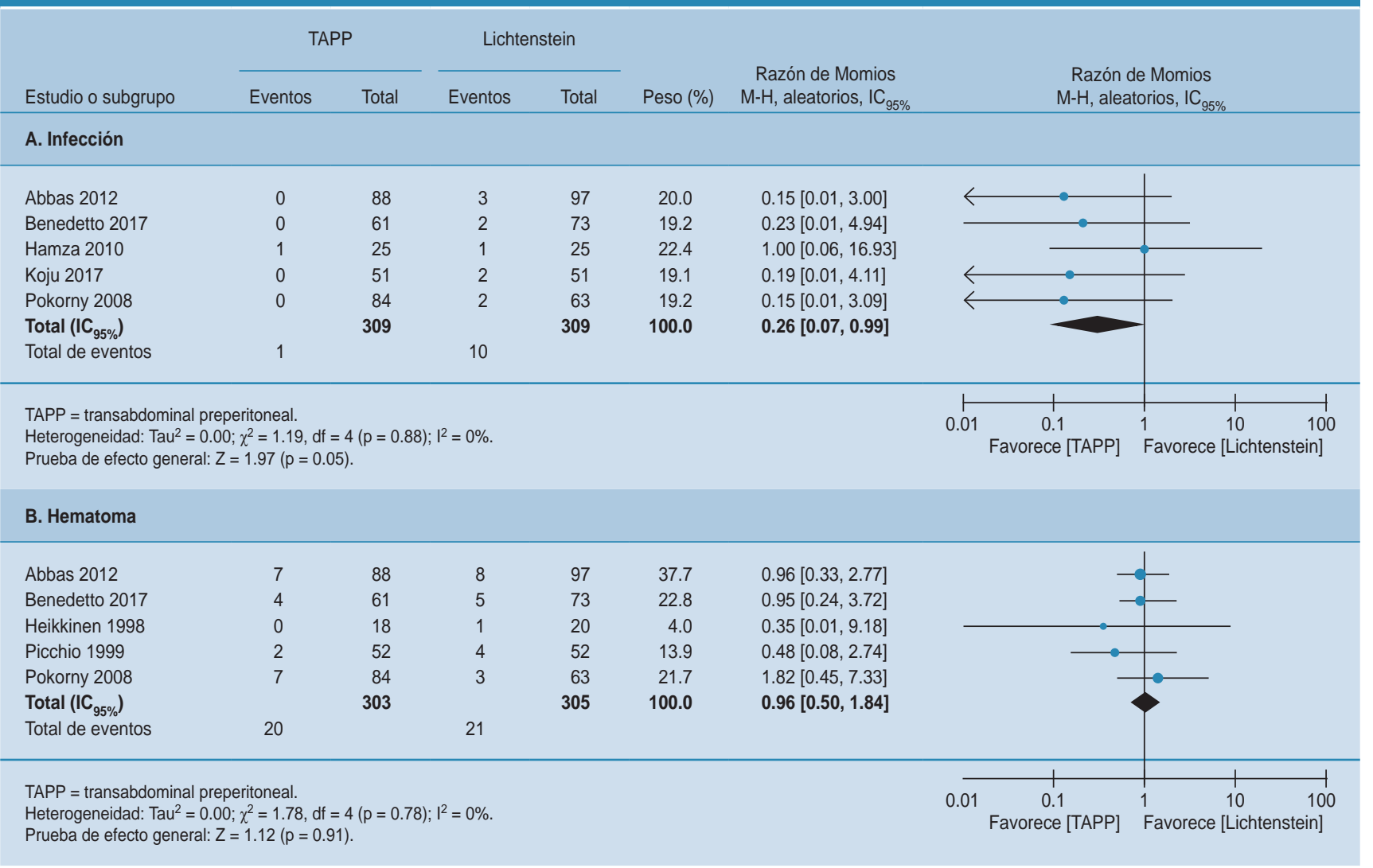

En lo que respecta a la duración de la cirugía, se establece que la técnica abierta tiene menor duración comparada con la técnica laparoscópica, en este análisis se observa una diferencia significativa en el tiempo. Estos resultados concuerdan con algunos estudios previos..$^{7-9,11}$ Se podría explicar por la menor dificultad técnica de la cirugía abierta y la complejidad de la técnica laparoscópica en condiciones no ideales de la cavidad (adherencias).

De los estudios que registraron el tiempo de hospitalización de los pacientes, no se encontró diferencia significativa entre las técnicas, algunos artículos refieren menor tiempo de hospitalización en el caso de TAPP, pero sin significancia. ${ }^{15,21}$

En el caso de infección de herida quirúrgica existieron más casos en la técnica abierta con significancia estadística. Aunque sólo cinco estudios reportan esta complicación.

El hematoma postquirúrgico se presentó en menos de $10 \%$ de los pacientes, no se encontró diferencia estadísticamente significativa entre los grupos.

No fue fácil el análisis del dolor agudo, la técnica para evaluarlo en cada artículo era distinta. Algunos utilizaban la escala análoga del dolor, otros la medicación e incluso algunos sólo un sistema binario, preguntando si había dolor o no. Además, el tiempo postquirúrgico en el que se evaluó el dolor difería en muchos artículos.

Respecto a los hallazgos, se puede observar una tendencia hacia menor presentación de dolor agudo en el grupo TAPP, encontrando una diferencia significativa en las primeras 12 horas postquirúrgicas. Los demás grupos analizados no mostraron diferencias estadísticamente significativas, pero sí una tendencia en favor del grupo TAPP.

En el caso de esta variable se debe tomar en consideración que la EVA es subjetiva, y depende del control analgésico que se le dé a cada paciente.

Se necesitan más ensayos clínicos aleatorizados para comparar estos abordajes, además deben contar con mayor control, registro y reporte de las variables y resultados. En este metaanálisis observamos algunas ventajas de la técnica laparoscópica TAPP frente a TL, principalmente en cuestión de dolor y complicaciones agudas, excepto en el caso del tiempo quirúrgico, el cual es menor en la técnica abierta.

La variabilidad del tiempo de seguimiento de cada estudio dificulta el análisis de las complicaciones crónicas a largo plazo. 
En el caso del dolor crónico se puede establecer que la técnica TAPP presenta menor riesgo frente a la TL. Se encontró una diferencia significativa en este estudio, lo que va acorde a estudios previos. ${ }^{11,28}$ En el caso de la técnica abierta se realiza una incisión mayor, además de la disección del cordón espermático y el músculo cremáster, lo que se podría asociarse a mayor dolor crónico, mientras que con la técnica TAPP existe una disección de peritoneo parietal que ocasiona una peritonitis local, relacionándose posiblemente con mayor dolor agudo postoperatorio.

La recurrencia de hernias inguinales se observó en más pacientes del grupo TAPP versus el TL, pero al realizar el análisis no se encontró diferencia estadísticamente significativa entre las técnicas. Es posible que sea necesario el estudio de una población mayor. La experiencia del

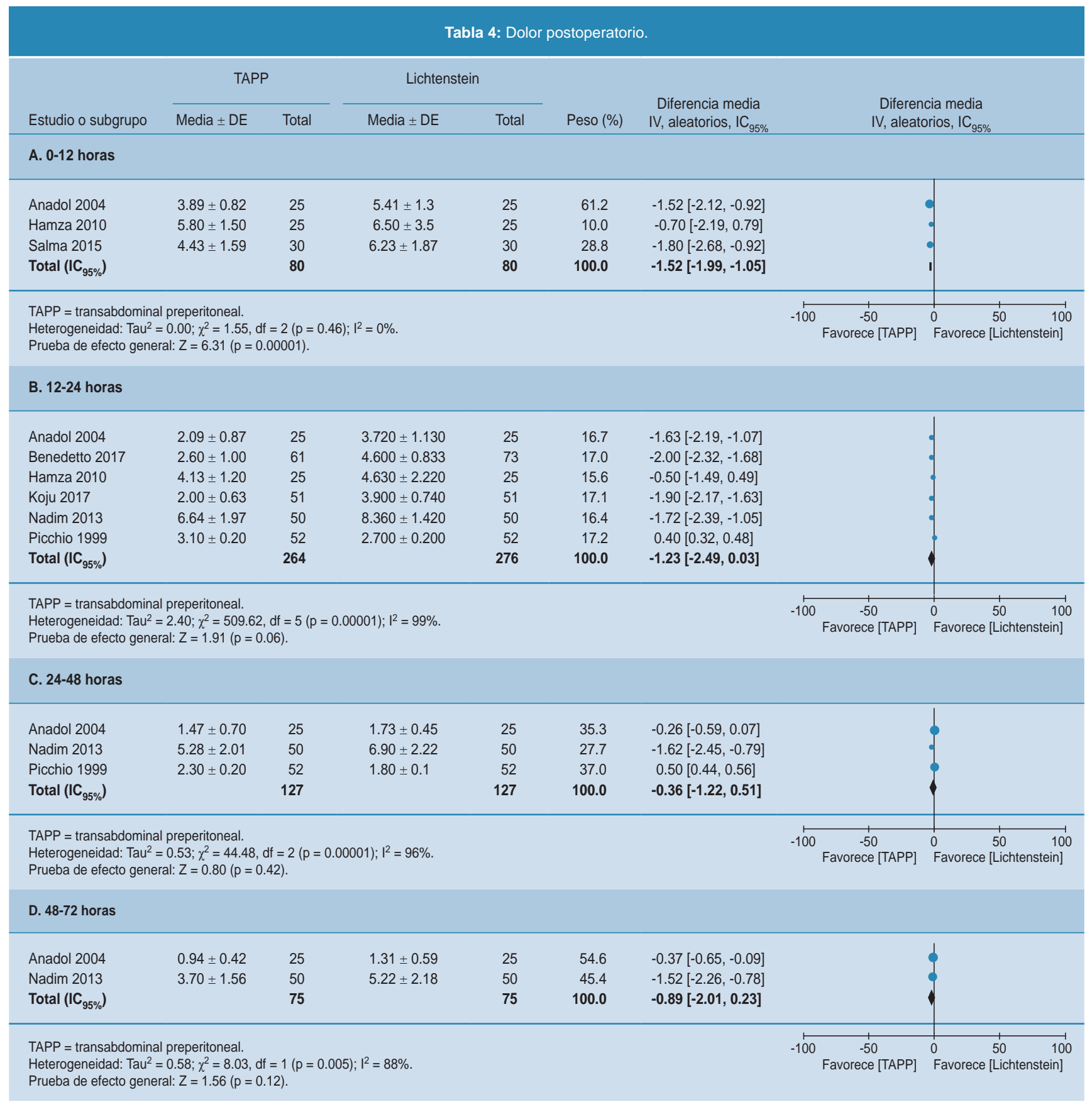


Tabla 5: Complicaciones crónicas.

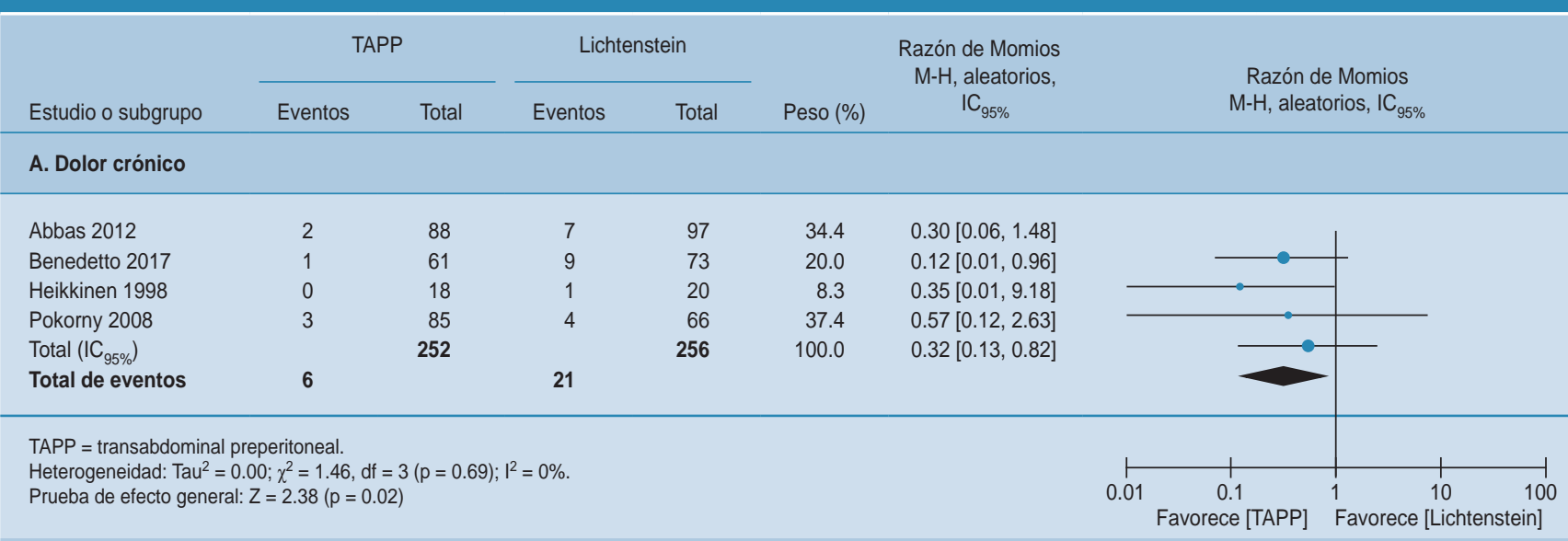

\section{B. Recurrencia}

$\begin{array}{lrrrrrl}\text { Abbas 2012 } & 3 & 88 & 5 & 97 & 28.5 & 0.65[0.15,2.80] \\ \text { Benedetto 2017 } & 4 & 61 & 4 & 73 & 29.8 & 1.21[0.29,5.06] \\ \text { Hamza 2010 } & 1 & 25 & 0 & 25 & 5.8 & 3.12[0.12,80.39] \\ \text { Koju 2017 } & 3 & 51 & 0 & 51 & 6.8 & 7.43[0.37,147.67] \\ \text { Nadim 2013 } & 3 & 50 & 6 & 50 & 29.1 & 0.47[0.11,1.99] \\ \text { Total (IC } & & & & & \\ \text { Toto }) & 14 & 15 & & & & \end{array}$

TAPP $=$ transabdominal preperitoneal

Heterogeneidad: Tau $^{2}=0.00 ; \chi^{2}=3.67, d f=4(p=0.45) ; I^{2}=0 \%$.

Prueba de efecto general: $Z=0.21(p=0.83)$.

cirujano y otros factores podrían estar relacionados con esta complicación.

Este metaanálisis es uno de los pocos que se han establecido en relación con la hernioplastia inguinal TAPP vs. Lichtenstein, por lo que la información de ensayos o estudios se vuelve compleja por la cantidad de variables presentes en los procedimientos. Si bien no existe diferencia significativa, este estudio aporta fundamentos para la comprensión de las variables de este tipo de procedimientos.

\section{REFERENCIAS}

1. Zaga MI. Actualidades en hernia inguinal. Estado del arte. Cir Gen. 2005; 27 (2): 164-167.

2. Gabrielli M. Resultados quirúrgicos de la hernioplastia inguinal laparoscópica con técnica transabdominal pre-peritoneal (TAPP). Rev Chil Cir. 2015; 67 (2): 167-174.

3. Gac EP, Uherel PF, Del Pozo LM, Oropesa CA, Rocco RE. Hernia inguinal: Un desafío quirúrgico permanente. Cuadernos de Cirugía. 2001; 15 (1): 96-106.

4. Adorno A, Monges L, Lezcano MJ, Cuenca O, Berdejo J, Ramírez J. Abordaje laparoscópico TAPP de las hernias inguinales. Experiencia inicial Hospital Nacional. Cir Parag. 2017; 41 (1): 14-17.

5. Mayagoitia G J. Estado actual de la hernioplastia inguinal laparoscópica en la práctica del cirujano general. Cirujano General. 2013; 35 (1): 28-30.
6. Sauerland S, Walgenbach M, Habermalz B, Seiler C, Miserez M. Laparoscopic versus open surgical techniques for ventral or incisional hernia repair. Cochrane Database Syst Rev. 2011; (3): CD007781.

7. Grant AM. EU Hernia Trialists Collaboration. Laparoscopic versus open groin hernia repair: Meta-analysis of randomized trials based on individual patient data. Hernia. 2002; 6 (1): 2-10.

8. Memon MA, Cooper NJ, Memon B, Memon MI, Abrams KR. Meta-analysis of randomized clinical trials comparing open and laparoscopic inguinal hernia repair. Br J Surg. 2003; 90 (12): 14791492.

9. Schmedt CG, Sauerland S, Bittner R. Comparison of endoscopic procedures vs Lichtenstein and other open mesh techniques for inguinal hernia repair: A meta-analysis of randomized controlled trials. Surg Endosc. 2005; 19 (2): 188-199.

10. McCormack K, Wake B, Perez J, Fraser C, Cook J, Mclntosh E et al. Laparoscopic surgery for inguinal hernia repair: Systematic review of effectiveness and economic evaluation. Health Technol Assess. 2005; 9 (14): 1-203, iii-iv.

11. Scheuermann U, Niebisch S, Lyros O, Jansen-Winkeln B, Gockel I. Transabdominal preperitoneal (TAPP) versus Lichtenstein operation for primary inguinal hernia repair - A systematic review and metaanalysis of randomized controlled trials. BMC Surg. 2017; 17 (1): 55.

12. Higgins JPT, Green S. Cochrane handbook for systematic reviews of interventions Version 5.1.0 [updated March 2011]. The Cochrane Collaboration, www.handbook.cochrane.org. Updated 2011.

13. Köninger JS, Oster M, Butters M. Management of inguinal hernia-a comparison of current methods. Chirurg. 1998; 69 (12): 1340-1344. 
14. Köninger J, Redecke J, Butters M. Chronic pain after hernia repair: a randomized trial comparing Shouldice, Lichtenstein and TAPP. Langenbecks Arch Surg. 2004; 389 (5): 361-365.

15. Butters M, Redecke J, Koninger J. Long-term results of a randomized clinical trial of Shouldice, Lichtenstein and transabdominal preperitoneal hernia repairs. Br J Surg. 2007; 94 (5): 562-565.

16. Moher D, Liberati A, Tetzlaff J, Altman DG, PRISMA Group. Preferred reporting items for systematic reviews and meta-analyses: The PRISMA statement. Int J Surg. 2010; 8 (5): 336-341.

17. Luo D, Wan X, Liu J, Tong T. Optimally estimating the sample mean from the sample size, median, mid-range, and/or mid-quartile range. Stat Methods Med Res. 2018; 27 (6): 1785-1805.

18. Abbas AE, Abd Ellatif ME, Noaman N, Negm A, El-Morsy G, Amin M et al. Patient-perspective quality of life after laparoscopic and open hernia repair: a controlled randomized trial. Surg Endosc. 2012; 26 (9): 2465-2470.

19. Anadol ZA, Ersoy E, Taneri F, Tekin E. Outcome and cost comparison of laparoscopic transabdominal preperitoneal hernia repair versus open Lichtenstein technique. J Laparoendosc Adv Surg Tech A. 2004; 14 (3): 159-163.

20. Ielpo B, Duran H, Diaz E, Fabra I, Caruso R, Malavé L et al. A prospective randomized study comparing laparoscopic transabdominal preperitoneal (TAPP) versus Lichtenstein repair for bilateral inguinal hernias. Am J Surg. 2017; 216 (1): 78-83.

21. Hamza Y, Gabr E, Hammadi H, Khalil R. Four-arm randomized trial comparing laparoscopic and open hernia repairs. Int J Surg. 2010; 8 (1): $25-28$
22. Heikkinen TJ, Haukipuro K, Hulkko A. A cost and outcome comparison between laparoscopic and Lichtenstein hernia operations in a day-case unit. A randomized prospective study. Surg Endosc. 1998; 12 (10): 1199-1203.

23. Koju R, Koju RB, Malla B, Dongol Y, Thapa LB. Transabdominal preperitoneal mesh repair versus Lichtenstein's hernioplasty. J Nepal Health Res Counc. 2017; 15 (2): 135-140.

24. Nadim K, Tariq SB, Munir A, Zahoor A, Liaqat AS. Outcome and cost comparison of laparoscopic transabdominal preperitoneal hernia repair versus open Lichtenstein technique. Journal of Postgraduate Medical Institute. 2013; 27 (3): 310-316.

25. Picchio M, Lombardi A, Zolovkins A, Mihelsons M, La Torre G. Tension-free laparoscopic and open hernia repair: randomized controlled trial of early results. World J Surg. 1999; 23 (10): 10041007; discussion 1008-9.

26. Pokorny H, Klingler A, Schmid T, Fortelny R, Hollinsky C, Kawji R et al. Recurrence and complications after laparoscopic versus open inguinal hernia repair: Results of a prospective randomized multicenter trial. Hernia. 2008; 12 (4): 385-389.

27. Salma U, Ahmed I, Ishtiaq S. PMC4641256; a comparison of postoperative pain and hospital stay between Lichtenstein's repair and laparoscopic transabdominal preperitoneal (TAPP) repair of inguinal hernia: a randomized controlled trial. Pak J Med Sci. 2015; 31 (5): 1062-1066.

28. Aasvang EK, Gmaehle E, Hansen JB, Gmaehle B, Forman JL, Schwarz $\mathrm{J}$ et al. Predictive risk factors for persistent postherniotomy pain. Anesthesiology. 2010; 112 (4): 957-969. 